\title{
Gestational hypertension as a subclinical preeclampsia in view of serum levels of angiogenesis-related factors
}

\author{
Chikako Hirashima ${ }^{1}$, Akihide Ohkuchi ${ }^{1,2}$, Kayo Takahashi ${ }^{1}$, Hirotada Suzuki ${ }^{1}$, Mika Yoshida ${ }^{1}$, \\ Takako Ohmaru$^{1}$, Kazuo Eguchi ${ }^{3}$, Haruko Ariga ${ }^{1}$, Shigeki Matsubara ${ }^{1}$ and Mitsuaki Suzuki ${ }^{1}$
}

It is controversial whether gestational hypertension (GH) and preeclampsia (PE) have the same pathophysiology. Our aim was to clarify whether the serum soluble fms-like tyrosine kinase 1 (sFlt-1)/placental growth factor (PIGF) ratio and levels of soluble endoglin (sEng) are different in women with GH and with PE. In women with GH (15 cases), hypertension preceding PE ( $\mathrm{h}-\mathrm{PE}, 10$ cases) and PE in which hypertension and proteinuria occurred simultaneously (si-PE, 36 cases), blood samples were collected after disease onset. The levels of $\log _{10}$ (sFIt-1/PIGF) in women with GH were significantly lower than in women with h-PE and si-PE ( $1.65 \pm 0.39$ vs. $2.22 \pm 0.35$ and $2.15 \pm 0.46)$. The levels of $\log _{10}$ sEng in women with GH were also significantly lower than in women with h-PE and si-PE $(1.51 \pm 0.43$ vs. $1.87 \pm 0.21$ and $1.85 \pm 0.32)$. The incidence rates of the sFIt-1/PIGF ratio $\geqslant 95$ th percentile of the reference value were 73,100 and $92 \%$, respectively, $(P=0.080)$, and those of sEng $\geqslant 95$ th percentile were 67,100 and $89 \%$, respectively, $(P=0.053)$. In conclusion, the levels of sFlt-1/PIGF ratio and sEng in women with GH were lower than in those with h-PE and with si-PE; however, the majority of women with GH showed abnormal increases of both sFIt-1/PIGF ratio and sEng, suggesting that GH may be a subclinical PE in view of serum levels of angiogenesis-related factors.

Hypertension Research (2011) 34, 212-217; doi:10.1038/hr.2010.212; published online 4 November 2010

Keywords: preeclampsia; gestational hypertension; soluble fms-like tyrosine kinase 1; placental growth factor; soluble endoglin

\section{INTRODUCTION}

Gestational hypertension $(\mathrm{GH})$ is hypertension without proteinuria occurring after the 20th week of gestation but resolving by the 12th week postpartum, ${ }^{1-3}$ whereas preeclampsia (PE) is defined as hypertension with proteinuria occurring after the 20th week of gestation but resolving by the 12th week postpartum. Therefore, the only difference between GH and PE is the appearance of proteinuria. ${ }^{1-3} \mathrm{New}$ hypertension in the second half of pregnancy (presumptive GH) progressed to $\mathrm{PE}$ in $15 \%,{ }^{4} 17 \%{ }^{5}$ in patients with mild GH remote from term, $46 \%$ ultimately had PE. ${ }^{6}$ Thus, not a few of presumptive GH change to PE. Therefore, GH and PE may have the same root preceding the onset of hypertension. However, it is controversial whether GH and PE have the same pathophysiology. ${ }^{7}$

Recently, it has been shown that two antiangiogenic peptides produced by the placenta, soluble fms-like tyrosine kinase 1 (sFlt-1) and soluble endoglin (sEng), contribute to the pathogenesis of PE. ${ }^{8-12}$ Although the mechanisms of sEng through which hypertension and proteinuria occur have not been clearly clarified, ${ }^{13}$ it is speculated that the increase of sFlt-1 weakens an action of placental growth factor (PIGF) or vascular endothelial growth factor-A, resulting in hypertension and proteinuria via endothelial injury. ${ }^{14,15}$ In comparative studies of serum levels of sFlt-1, sFlt-1/PlGF ratio, sEng in women with GH and PE, the mean serum levels of sFlt-1, sFlt-1/PlGF ratio and sEng in women with $\mathrm{PE}$ were significantly higher than in women with $\mathrm{GH}$; those in women with GH were higher than in control women. ${ }^{10,16}$ Therefore, mild increases of sFlt-1, sFlt-1/PIGF ratio and sEng may be characteristics of $\mathrm{GH}$, whereas severe increases of these three markers may be characteristics of PE.

Although PE includes the following three conditions: that is, proteinuria preceding $\mathrm{PE}$ ( $\mathrm{p}-\mathrm{PE}$ ), hypertension preceding PE (h-PE) and $\mathrm{PE}$ with almost simultaneous onsets of hypertension and proteinuria (si-PE), ${ }^{5}$ the current definition of pregnancy-induced hypertension does not discriminate these three conditions. ${ }^{1-3}$ We thought that the comparison between GH and h-PE might break the ice for elucidating the differences of pathophyisology between GH and PE. We aimed to compare serum levels of sFlt-1, PlGF, sFlt-1/PlGF and sEng among women with GH, h-PE and si-PE. 


\section{METHODS}

\section{Subjects and procedures}

We obtained written informed consent from all women as well as the approval of the Ethics Committee of our institute. The study subjects consisted of two groups: one for constructing reference ranges of serum sFlt-1, PlGF and sFlt-1/PlGF ratio according to gestational age and the other for evaluating the serum sFlt-1, PlGF, sFlt-1/PlGF ratio and sEng levels after the clinical manifestation of GH, h-PE and si-PE. The study subjects were all Japanese women with singleton pregnancies. The first group consisted of 82 pregnant controls without $\mathrm{PE}$ or $\mathrm{GH}$, from whom blood samples were collected three times at 20-23, 27-30 and 36-38 weeks of gestation; the samples were serially collected between April 2004 and April 2007; these samples were previously used for constructing reference ranges of serum sEng. ${ }^{12}$ The second group consisted of 15 women with GH, $10 \mathrm{~h}-\mathrm{PE}$ and 36 si-PE, from whom blood samples were collected as soon as possible after admission to hospital due to the clinical manifestation of GH or PE between April 2000 and April 2007; in this study, we could not obtain samples before the occurrence of $\mathrm{PE}$ in women with h-PE; we previously used the 46 samples of PE for the analysis of sEng. ${ }^{12}$

Blood samples were centrifuged at $4{ }^{\circ} \mathrm{C}$ at 2500 r.p.m. for $15 \mathrm{~min}$. Samples were stored at $-20^{\circ} \mathrm{C}$ until use. Enzyme-linked immunosorbent assays for human sFlt-1 (DVR100B, R\&D Systems, Minneapolis, MN, USA), PlGF (DPG00, R\&D systems) and sEng (DNDG00, R\&D systems) were performed in duplicate according to the manufacturer's instructions. The minimal detectable dose in the assays for sFlt-1, PlGF and sEng were 3.5, 7 and $7 \mathrm{pg} \mathrm{ml}^{-1}$, respectively. The intraassay and interassay coefficients of variation for sFlt-1 were 2.6-3.8 and 5.5-9.8\%, respectively; those for PlGF were 3.6-7.0 and $10.9-11.8 \%$, respectively; and those for sEng were $2.8-3.2$ and $6.3-6.7 \%$, respectively.

\section{Definitions}

We defined GH and PE according to the definition and classification of pregnancy-induced hypertension (2004) of the Japan Society for the Study of Hypertension in Pregnancy. ${ }^{1}$ In brief, GH was defined as hypertension without proteinuria occurring after the 20th week of gestation but resolving by the 12th week of postpartum. PE was defined as hypertension with proteinuria occurring after the 20th week of gestation but resolving by the 12th week of postpartum. Superimposed PE includes the following three conditions: (1) chronic hypertension diagnosed before pregnancy or to the 20th week of gestation, with which proteinuria emerges after the 20th week of gestation; (2) aggravations of either or both hypertension and proteinuria in women with preexisting hypertension and proteinuria after the 20th week of gestation; (3) hypertension emerging after the 20th week of gestation in patients with preexisting renal diseases that manifest solely proteinuria. In this study, we defined de novo hypertension without proteinuria occurring after the 20th week of gestation as presumptive $\mathrm{GH}$. We also divided $\mathrm{PE}$ into three conditions: $\mathrm{p}-\mathrm{PE}, \mathrm{h}-\mathrm{PE}$ and PE with si-PE. H-PE includes the following two conditions, chronic hypertension preceding $\mathrm{PE}$ and presumptive $\mathrm{GH}$ preceding PE. Hypertension was defined when systolic blood pressure level was equal to or over $140 \mathrm{~mm} \mathrm{Hg}$ and/or diastolic blood pressure level was equal to or over $90 \mathrm{~mm} \mathrm{Hg}$. Proteinuria was defined as $300 \mathrm{mg}$ per day from $24 \mathrm{~h}$ urine collection. If only test tape was available, repeated semiquantitative test results of $1+$, which represent $30 \mathrm{mg}$ per $100 \mathrm{ml}$ of protein or more, were considered to constitute a positive result. We diagnosed women with the following three findings-serum lactate dehydrogenase levels $>1.5$-fold the upper limit in our institute, serum aspartate aminotransferase levels $>1.5$-fold the upper limit in our institute and low platelets (platelet count $<10 \times 10^{4} \mu \mathrm{l}^{-1}$ )—as having hemolysis, elevated liver enzyme and low platelet (HELLP) syndrome, respectively. In this study, we included partial HELLP syndrome in the category of HELLP syndrome; we diagnosed women with two but not all the three features of HELLP syndrome as having partial HELLP syndrome. We defined an small-for-gestational-age (SGA) infant as having a birth weight below the 10th percentile for the Japanese population. ${ }^{17}$ The multiple of the median for birth weight was calculated as (A-B)/B (where $\mathrm{A}$ is the infant's birth weight and $\mathrm{B}$ is the median birth weight at the corresponding gestational week), representing the degree of deviation from the median.

\section{Constructions of normal reference values of sFlt-1, PIGF and sFlt-1/PlGF ratio}

In 82 women, serial serum data were examined throughout the second half of pregnancy. These data had been used for constructing normal reference values of sEng throughout the second half of pregnancy. ${ }^{12}$ The data distributions of $\log _{10}$ sFlt-1, $\log _{10}$ PlGF and $\log _{10}$ (sFlt-1/PlGF) each week were close to normal distribution using normal proportion-proportion plots when the sample size each week was at least 10 . The changes from 20 to 38 weeks of gestation of $\log _{10}$ SFlt-1, $\log _{10} \mathrm{PlGF}$ and $\log _{10}($ sFlt-1/PlGF $)$ approximated quadratic curves (Figures $1 \mathrm{a}, \mathrm{b}$ and $\mathrm{c}$ ).
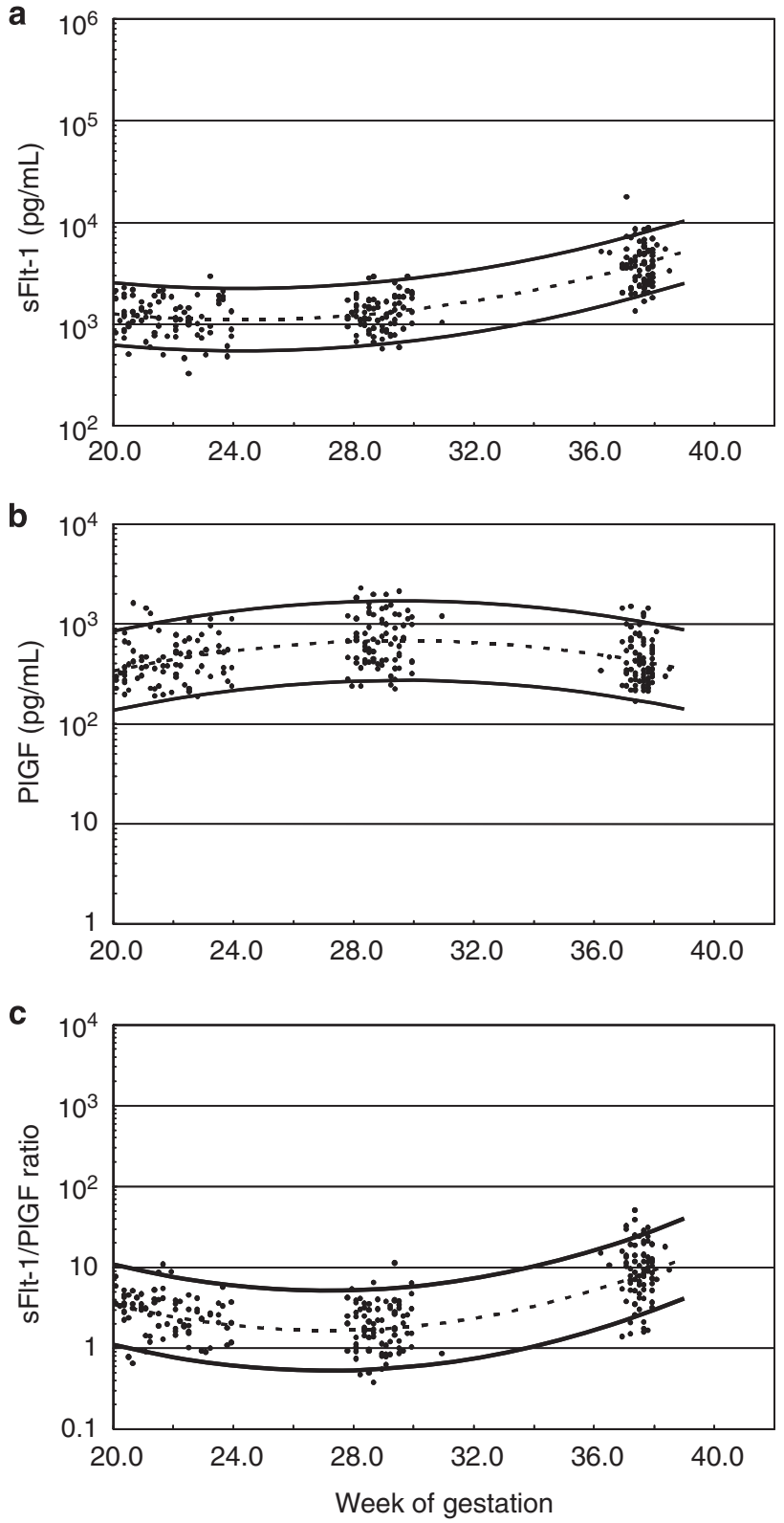

Figure 1 Quadratic curves representing the mean and the 5th and 95th percentiles of the serum level of sFlt-1 (a), PIGF (b) and the sFIt-1/PIGF ratio (c) from 20 to 38 weeks of gestation. Solid curves represent the 5th and 95th percentiles of the reference values, and the dotted curve represents the mean. Closed circles represent the 82 women examined in this study. The means each week could be represented by quadratic curves, and the standard deviation scores each week could be represented by constant values. 
The distribution of $\log _{10}$ SFlt-1 during the second half of pregnancy could be represented by a quadratic curve $\left(y=0.00308 x^{2}-0.1496 x+4.860, y\right.$ : mean $\log _{10}$ sFlt-1, $x$ : weeks of gestation, $\left.P<0.001\right)$. We hypothesized that the middle value each week on the quadratic curve was equal to the mean of $\log _{10} \mathrm{SFlt}-1$ each week. As the result of Levene statistics of $\log _{10}$ SFlt-1 at 20-38 weeks was not significant $(P=0.069)$, we hypothesized that the s.d. of $\log _{10}$ sFlt-1 each week was constant. The absolute values of the residuals were plotted individually against weeks and fitted by a linear curve, revealing that the constant value was 0.149 . The s.d. was calculated by multiplying the constant value by the root of $\pi / 2,18$ and was 0.187 . Thus, the 95 th percentile of $\log _{10}$ sFlt- 1 was defined as mean $\log _{10}$ sFlt- $1+1.645$ s.d. $\left(y=0.00308 x^{2}-0.1496 x+5.168, y\right.$ : 95th percentile of $\log _{10}$ sFlt-1, $x$ : weeks of gestation) (Figure 1a).

The distribution of $\log _{10} \mathrm{PlGF}$ during the second half of pregnancy could be represented by a quadratic curve $\left(y=-0.00326 x^{2}+0.1930 x-0.0228, y\right.$ : mean $\log _{10}$ PlGF, $x$ : weeks of gestation, $\left.P<0.001\right)$. As the result of Levene statistics of $\log _{10} \mathrm{PlGF}$ was not significant $(P=0.233)$, we hypothesized that the s.d. of $\log _{10} \mathrm{PlGF}$ each week was constant. Using the same method, which determined the s.d. of $\log _{10}$ sFlt-1, we calculated the s.d. of $\log _{10} \mathrm{PlGF}$ to be 0.241 . Thus, the 5 th percentile of $\log _{10} \mathrm{PlGF}$ was defined as mean $\log _{10} \mathrm{PlGF}-1.645$ s.d. $\left(y=-0.00326 x^{2}+0.1930 x-0.419, y\right.$ : 5 th percentile of $\log _{10}$ PlGF, $x$ : weeks of gestation) (Figure 1b).

The distribution of $\log _{10}(\mathrm{sFlt}-1 / \mathrm{PlGF})$ during the second half of pregnancy could be represented by a quadratic curve $\left(y=0.00633 x^{2}-0.3436 x+4.882, y\right.$ : mean $\log _{10}$ (sFlt-1/PlGF), $x$ : weeks of gestation, $\left.P<0.001\right)$. Although the result of Levene statistics of $\log _{10}(\mathrm{sFlt}-1 / \mathrm{PlGF})$ was significant $(P=0.022)$, there was no tendency of an increase or decrease according to gestational age. Therefore, we hypothesized that the s.d. of $\log _{10}(\mathrm{sFlt}-1 / \mathrm{PlGF})$ each week was also constant. Using the same method, which determined the s.d. of $\log _{10}$ sFlt-1, we calculated the s.d. of $\log _{10}(\mathrm{sFlt}-1 / \mathrm{PlGF})$ to be 0.301 . Thus, the 95 th percentile of $\log _{10}(\mathrm{sFlt}-$ $1 / \mathrm{PlGF})$ was defined as mean $\log _{10}(\mathrm{sFlt}-1 / \mathrm{PlGF})+1.645$ s.d. $(y=0.00633$ $x^{2}-0.3436 x+5.377, y$ : 95th percentile of $\log _{10}(\mathrm{sFlt}-1 / \mathrm{PlGF}), x$ : weeks of gestation) (Figure 1c).

When the raw values of normal pregnant women were plotted on the gestational age-specific normal reference curves of sFlt-1, PlGF and the sFlt-1/ PlGF ratio, most data were between the 5th and the 95th percentile curves, and there were no extremely biased values outside both the 5th and 95th percentiles curves, indicating that the calculated gestational age-specific normal reference curves of sFlt-1, PlGF and the sFlt-1/PlGF ratio appropriately represented the distributions of real values.

\section{Statistics}

We calculated the standard deviation scores of $\log _{10}$ Flt-1, $\log _{10} \mathrm{PlGF}, \log _{10}$ (sFlt-1/PlGF) and $\log _{10}$ sEng and as (A-B)/C (where $\mathrm{A}$ is the raw value, $\mathrm{B}$ is the mean and $C$ is the s.d.). We defined high sFlt1, the sFlt-1/PlGF ratio and sEng as concentrations $\geqslant 95$ th percentile of the reference values at a given gestational week; low PlGF as a concentration $<5$ th percentile of the reference value at a given gestational week. The results are presented as the mean \pm s.d. For serum sFlt-1, PlGF, sFlt-1/PlGF ratio and sEng levels, statistical testing was conducted after logarithmic transformation because it was proven that all four indices of angiogenesis-related factors after logarithmic transformation in pregnant controls closely resembled normal distributions using normal proportion-proportion plots and the Kolmogonov-Smirnov test. The chi-square test or Fisher's exact test was used to compare the categorical data. For multiple group comparisons, homogeneity of variance was assessed using the Levene test. We used one way analysis of variance to test for overall differences among groups, followed by Gabriel's method to compare separate group means when the Levene test was not significant and followed with the Dunnett-T3 method for comparison when the Levene test was significant. The fitted curve estimation was performed using built-in software within the SPSS software package (version 13.0J for Windows). $P<0.05$ was considered statistically significant.

\section{RESULTS}

Women with GH gave birth to infants $\sim 3-4$ weeks later than those with PE (Table 1). Women with GH had preterm delivery less than those with h-PE and si-PE, respectively; and the infant birth weight in women with GH was much larger than in those with h-PE and with si-PE. The incidence rates of an SGA infant were not different among $\mathrm{GH}, \mathrm{h}-\mathrm{PE}$ and si-PE; however, the multiple of the median of the infant birth weight in women with GH was larger than in women with h-PE and si-PE, although the differences did not reach significance. There were no significant differences in the frequencies of nulliparity, BP $\geqslant 160 / 110 \mathrm{~mm} \mathrm{Hg}$, HELLP syndrome, eclampsia and the levels of systolic blood pressure/diastolic blood pressure.

$\mathrm{GH}, \mathrm{h}-\mathrm{PE}$ and si-PE all affected the serum sFlt-1, PlGF, sFlt-1/PlGF ratio and sEng levels (Figure 2); however, the levels of sFlt-1, sFlt-1/ PlGF ratio and sEng in women with GH were significantly lower than in those with h-PE and with si-PE (Table 2). The levels of PlGF in

Table 1 Maternal and infantile characteristics in women with GH, h-PE and si-PE

\begin{tabular}{|c|c|c|c|c|c|}
\hline & $\mathrm{GH}$ (group 1) $(\mathrm{n}=15)$ & $h-P E($ group 2$)(\mathrm{n}=10)$ & si-PE (group 3) $(\mathrm{n}=36)$ & $\mathrm{P}$-value & Significant pairs \\
\hline Age (years) & $33.4 \pm 4.8$ & $32.2 \pm 5.4$ & $32.1 \pm 5.4$ & 0.725 & \\
\hline Body height $(\mathrm{m})$ & $1.57 \pm 0.04$ & $1.60 \pm 0.06$ & $1.58 \pm 0.06$ & 0.512 & \\
\hline Prepregnancy body weight (kg) & $62.0 \pm 12.9$ & $65.3 \pm 10.5$ & $55.8 \pm 10.1$ & 0.029 & 2 vs. 3 \\
\hline Prepregnancy BMI (kg m${ }^{-2}$ ) & $25.0 \pm 5.2$ & $25.6 \pm 4.0$ & $22.5 \pm 4.0$ & 0.056 & \\
\hline Nullipara (\%) & $12(80 \%)$ & $7(70 \%)$ & $22(61 \%)$ & 0.416 & \\
\hline Gestational age at onset (weeks) & $35.6 \pm 4.8$ & $31.3 \pm 2.9$ & $32.3 \pm 4.5$ & 0.146 & \\
\hline $\mathrm{BP} \geqslant 160 / 110 \mathrm{~mm} \mathrm{Hg}(\%)$ & $12(80 \%)$ & $9(90 \%)$ & $27(75 \%)$ & 0.586 & \\
\hline Maximum SBP (mm Hg) & $167 \pm 7$ & $180 \pm 15$ & $171 \pm 16$ & 0.068 & \\
\hline Maximum DBP $(\mathrm{mm} \mathrm{Hg})$ & $103 \pm 13$ & $108 \pm 7$ & $105 \pm 10$ & 0.546 & \\
\hline Urinary protein $\geqslant 2$ g per day $(\%)$ & - & $4(40 \%)$ & $17(47 \%)$ & 0.745 & \\
\hline HELLP syndrome $(\%)^{\mathrm{b}}$ & $1(6.7 \%)$ & $1(10 \%)$ & $7(19 \%)$ & 0.452 & \\
\hline Eclampsia (\%) & $0(0 \%)$ & $0(0 \%)$ & $1(2.8 \%)$ & 0.703 & \\
\hline Gestational age at delivery (weeks) & $36.7 \pm 3.4$ & $32.6 \pm 3.0$ & $33.7 \pm 4.3$ & 0.021 & 1 vs. 2,1 vs. 3 \\
\hline Preterm delivery (\%) & $6(40 \%)$ & $10(100 \%)$ & $28(78 \%)$ & 0.002 & 1 vs. 2,1 vs. 3 \\
\hline Infant birth weight (g) & $2477 \pm 862$ & $1469 \pm 581$ & $1761 \pm 823$ & 0.005 & 1 vs. 2,1 vs. 3 \\
\hline Infant birth weight (MoM) & $-0.03 \pm 0.20$ & $-0.20 \pm 0.15$ & $-0.15 \pm 0.18$ & 0.050 & \\
\hline Small-for-gestational-age infants (\%) & $4(27 \%)$ & $4(40 \%)$ & $19(53 \%)$ & 0.222 & \\
\hline
\end{tabular}

Abbreviations: BMI, body mass index; BP, blood pressure; DBP, diastolic BP; GH, gestational hypertension; HELLP, hemolysis, elevated liver enzyme, and low platelet; h-PE, hypertension preceding preeclampsia; MoM, the multiple of the median; SBP, systolic BP; si-PE, almost simultaneous onset of both hypertension and proteinuria. preeclampsia; MoM, the multiple of the median; SBP, systolic BP; Si-PE, almost simultaneous onset of both hypertension and proteinuria.
aln women with GH, the onset of GH was defined as the gestational weeks when hypertension occurred. In women with PE, the onset of PE was defined as the gestational weeks when both

bPartial HELLP syndrome was included in this category.
bypertension and roteinuria occurred. 

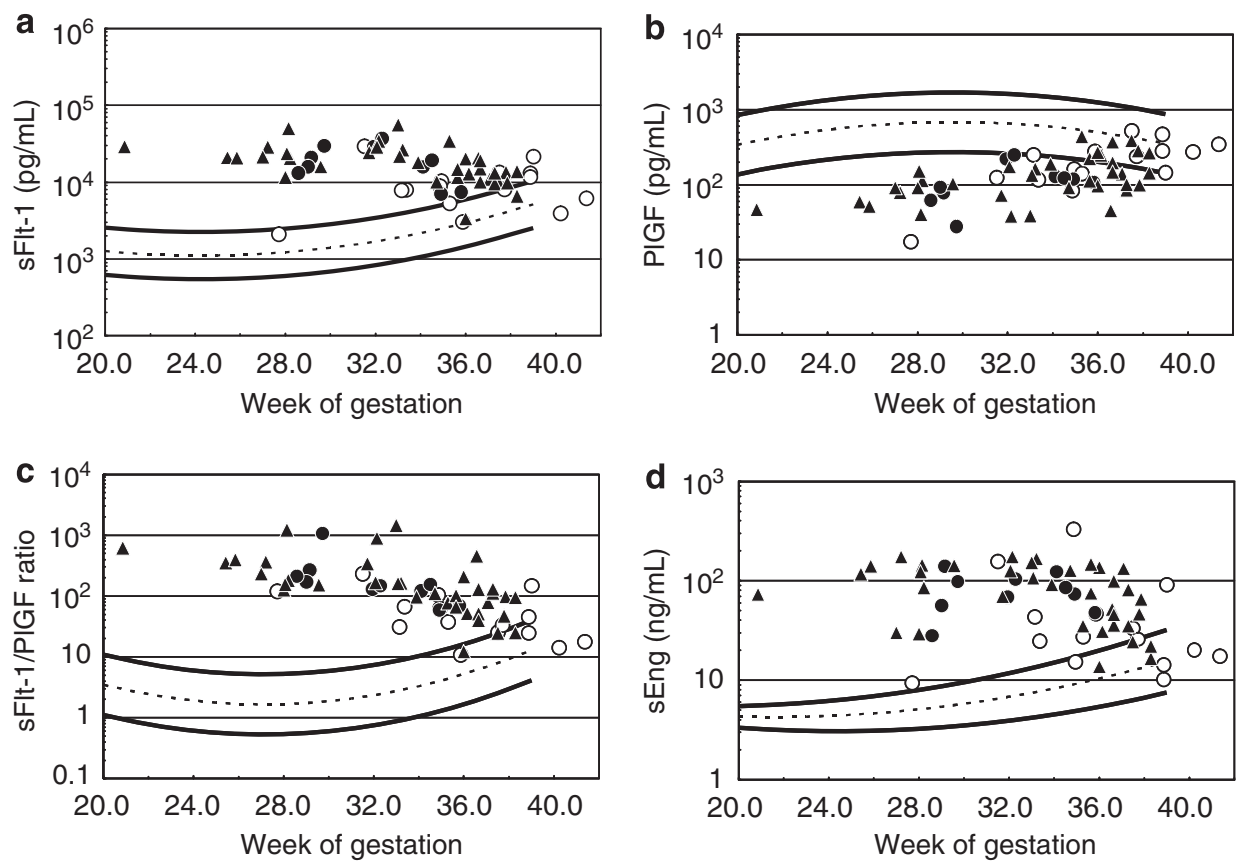

Figure 2 Serum levels of sFIt-1 (a), PIGF (b), sFlt-1/PIGF ratio (c) and sEng (d) in 15 women with GH, 10 women with h-PE and 36 women with si-PE were plotted against gestational age-specific normal reference values. Blood samples were collected as soon as possible after the clinical manifestation of GH or PE. Solid curves represent the 5th and 95th percentiles of reference values, and dotted curves represent the means. Open circles, data in women with GH $(n=15)$. Closed circles, data in women with h-PE $(n=10)$. Closed triangles, data in women with si-PE $(n=36)$.

Table 2 Serum levels of sFIt-1, PIGF, the sFIt-1/PIGF ratio and sEng in women with GH, h-PE and si-PE

\begin{tabular}{|c|c|c|c|c|c|}
\hline Gestational age at measurement (weeks) & GH (group 1) $(\mathrm{n}=15)$ & $h-P E($ group 2) $(\mathrm{n}=10)$ & si-PE (group 3) $(\mathrm{n}=36)$ & P-value & Significant pairs \\
\hline $\log _{10}$ sFlt-1 $\left(\mathrm{pg} \mathrm{ml}^{-1}\right)$ & $3.91 \pm 0.30$ & $4.24 \pm 0.24$ & $4.23 \pm 0.24$ & $<0.001$ & 1 vs. 2,1 vs. 3 \\
\hline sFlt-1 $\geqslant 95$ th percentile $(\%)$ & $10(67 \%)$ & $10(100 \%)$ & $34(94 \%)$ & 0.008 & 1 vs. 3 \\
\hline \multicolumn{6}{|l|}{ PIGF } \\
\hline PIGF < 5th percentile (\%) & $6(40 \%)$ & $10(100 \%)$ & $27(75 \%)$ & 0.004 & 1 vs. 2,1 vs. 3 \\
\hline \multicolumn{6}{|l|}{ sFIt-1/PIGF ratio } \\
\hline $\log _{10}(\mathrm{sFIt}-1 / \mathrm{PIGF})$ & $1.65 \pm 0.39$ & $2.22 \pm 0.35$ & $2.15 \pm 0.46$ & $<0.001$ & 1 vs. 2,1 vs. 3 \\
\hline $\log _{10}(\mathrm{sFIt}-1 / \mathrm{PIGF}) \mathrm{SDS}$ & $2.84 \pm 2.21$ & $6.01 \pm 1.61$ & $5.23 \pm 2.21$ & $<0.001$ & 1 vs. 2,1 vs. 3 \\
\hline sFIt-1/PIGF ratio $\geqslant 95$ th percentile $(\%)$ & $11(73 \%)$ & $10(100 \%)$ & $33(92 \%)$ & 0.080 & \\
\hline \multicolumn{6}{|l|}{ SEng } \\
\hline sEng $\geqslant 95$ th percentile $(\%)$ & $10(67 \%)$ & $10(100 \%)$ & $32(89 \%)$ & 0.054 & \\
\hline
\end{tabular}

Abbreviations: GV, gestational hypertension; h-PE, hypertension preceding preeclampsia; PIGF, placental growth factor; SDS, standard deviation score; sEng, soluble endoglin; sFIt-1, soluble fmslike tyrosine kinase 1 ; si-PE, almost simultaneous onset of both hypertension and proteinuria.

women with GH were higher than in those with h-PE and with si-PE, although the differences did not reach significance. Thus, the changes of angiogenesis-related factors in women with $\mathrm{GH}$ were milder than in those with h-PE and si-PE. The deviation of the four indices of angiogenesis-related factors in women with GH were significantly smaller than in those with h-PE and with si-PE, except for the levels of PlGF standard deviation scores between women with GH and with
si-PE. However, the rates of high sFlt-1/PlGF ratio and high sEng were not significantly different among these three groups; high sFlt-1/PlGF ratio and high sEng were seen in $>2 / 3$ in women with $\mathrm{GH}$, whereas $100 \%$ in those with h-PE and si-PE.

We examined whether preterm birth affected the serum levels of sFlt-1, PlGF, sFlt-1/PlGF ratio and sEng in women with GH and PE. In $\mathrm{GH}$ women with preterm birth, the level of $\log _{10} \mathrm{PlGF}$ was 
significantly lower than in those with term birth $(1.99 \pm 0.16 v s$. $2.44 \pm 0.07, P=0.012)$, and the level of $\log _{10}($ sFlt-1/PlGF $)$ was significantly higher than in those with term birth $(1.93 \pm 0.12$ vs. $1.46 \pm 0.11$, $P=0.015$ ), whereas the levels of $\log _{10}$ sFlt -1 and $\log _{10}$ sEng were not significantly different between GH women with preterm birth and those with term birth $(3.92 \pm 0.15$ vs. $3.90 \pm 0.09 ; 1.65 \pm 0.24 v s$. $1.41 \pm 0.09$, respectively). In PE women with preterm birth, the levels of $\log _{10}$ sFlt-1, $\log _{10}$ (sFlt-1/PlGF) and $\log _{10}$ sEng were significantly higher than in those with term birth $(4.31 \pm 0.03$ vs. $4.04 \pm 0.04$, $P=0.001 ; \quad 2.23 \pm 0.06$ vs. $1.86 \pm 0.09, \quad P=0.020 ; 1.91 \pm 0.04$ vs. $1.63 \pm 0.09, P=0.004$, respectively), whereas the level of $\log _{10} \mathrm{PlGF}$ was not significantly different between PE women with preterm birth and those with term birth $(2.08 \pm 0.05 v s .2 .17 \pm 0.07)$.

We also examined whether an SGA infant affected the serum levels of sFlt-1, PlGF, sFlt-1/PlGF ratio and sEng in women with GH and PE. In GH women with an SGA infant, the level of $\log _{10} \mathrm{PlGF}$ was significantly lower than in those without an SGA infant $(1.93 \pm 0.25$ vs. $2.38 \pm 0.06, P=0.025$ ), whereas the levels of $\log _{10}$ sFlt- $1, \log _{10}$ (sFlt1/PlGF) and $\log _{10}$ sEng were not significantly different between GH women with an SGA infant and without an SGA infant $(3.83 \pm 0.25$ vs. $3.94 \pm 0.07 ; 1.91 \pm 0.26$ vs. $1.56 \pm 0.09 ; 1.75 \pm 0.36$ vs. $1.42 \pm 0.08$, respectively). In PE women with an SGA infant, the level of $\log _{10} \mathrm{PlGF}$ was significantly lower than in those without an SGA infant (1.96 \pm 0.05 vs. $2.22 \pm 0.05, P=0.001$ ), and the levels of $\log _{10}$ sFlt 1 and $\log _{10}(\mathrm{sFlt}-1 / \mathrm{PlGF})$ were significantly higher than in those without an SGA infant (4.34 \pm 0.04 vs. $4.18 \pm 0.05, P=0.009 ; 2.38 \pm 0.08$ vs. $1.97 \pm 0.07, P<0.001$, respectively), whereas the level of $\log _{10}$ sEng was not significantly different between PE women with an SGA infant and without an SGA infant $(1.90 \pm 0.05$ vs. $1.83 \pm 0.05)$.

As an SGA infant affected the serum levels of PlGF, sFlt-1 or sFlt-1/ PlGF ratio in women with GH and PE, we also examined whether placental weight, which is mostly smaller and lighter in an SGA infant than in an appropriate-for-gestational-age infant, affected the serum levels of sFlt-1, PlGF, sFlt-1/PlGF ratio and sEng in normal pregnant women, women with GH and women with PE. An SGA significantly affected the placental weight in women with $\mathrm{PE}$, but not in normal pregnant women and women with GH. In normal pregnant women, placental weight was significantly positively correlated with the level of $\log _{10} \mathrm{PlGF}(r=0.290, P=0.008)$, but not $\log _{10}$ sFlt-1, $\log _{10}(\mathrm{sFlt}-1 / \mathrm{PlGF})$ and $\log _{10}$ sEng at 36-38 weeks of gestation. In women with $\mathrm{GH}$, placental weight was significantly positively correlated with the level of $\log _{10}$ PlGF $(r=0.777, P<0.001)$ and $\log _{10}$ sFlt-1 $(r=0.532, P=0.041)$, but not $\log _{10}(\mathrm{sFlt}-1 / \mathrm{PlGF})$ and $\log _{10}$ sEng. In women with $\mathrm{PE}$, placental weight was significantly positively correlated with the level of $\log _{10} \mathrm{PlGF}(r=0.654, P<0.001)$, but was significantly inversely correlated with $\log _{10}$ sFlt-1 $(r=-0.384, P=0.003), \log _{10}$ (sFlt-1/PlGF $)$ $(r=-0.661, P<0.001)$ and $\log _{10}$ sEng $(r=-0.382, P=0.003)$.

\section{DISCUSSION}

This study for the first time compared the levels of four indices of angiogenesis-related factors, that is, sFlt-1, PlGF, sFlt-1/PlGF ratio and sEng, among women with GH, h-PE and si-PE. We found that the sFlt-1/PlGF ratio and sEng deviated from normal reference values in the majority of women with $\mathrm{GH}$ and with $\mathrm{PE}$, although they were lower in women with GH than in women with h-PE or si-PE. These results suggested that $\mathrm{GH}$ may be a subclinical $\mathrm{PE}$ in view of serum levels of angiogenesis-related factors.

In our study, the levels of sFlt-1, the sFlt-1/PlGF ratio and sEng in women with GH were less than in those with PE. Our results are consistent with the previous data. ${ }^{10,16}$ Salahuddin et al. ${ }^{16}$ reported that the serum levels of both sFlt-1 and sEng in women with GH were significantly lower than in those with PE. In the study of Levine et al., ${ }^{10}$ women with GH showed an increased sFlt-1/PlGF ratio and sEng before the onset of GH similar to PE, although the deviations in the levels of the sFlt-1/PlGF ratio and sEng in women with GH were less than in preterm PE and term PE; in addition, the time when both indices increased was closer to the onset of disease in women with $\mathrm{GH}$ than in those with preterm PE or term PE. These evidences and our results suggest that the measurements of sFlt-1, sFlt-1/PlGF and sEng may be useful in differentiating PE from GH.

Our study for the first time compared the levels of four indices of angiogenesis-related factors, that is, sFlt-1, PlGF, sFlt-1/PlGF ratio and sEng, among women with GH, h-PE and si-PE. The changes of angiogenesis-related factors in women with $\mathrm{GH}$ were milder than in those with h-PE and si-PE. The deviations from the normal reference values of the four indices of angiogenesis-related factors in women with GH were smaller than in those with h-PE and with si-PE. However, the rates of high sFlt-1/PlGF ratio and high sEng were not significantly different among these three groups. In addition, high sFlt-1/PlGF ratio and high sEng were seen in $>2 / 3$ in women with $\mathrm{GH}$, whereas $100 \%$ in those with h-PE and si-PE. Therefore, we speculate that the majority of presumptive GH are at early and mild stages of subclinical PE, which change to symptomatic PE with the deteriorations of angiogenesis-related factors.

In view of pathology, $\mathrm{GH}$ and $\mathrm{PE}$ may be a continuous process rather than a separate abnormal condition. Strevens et al. ${ }^{19}$ obtained renal biopsy samples in women with normal pregnancy, GH and PE; glomerular endotheliosis, which has been believed as a characteristic lesion found in women with PE, was present in all women with GH and PE, and almost half of the controls, although the degree of endotheliosis is more severe in women with $\mathrm{PE}$ than in women with $\mathrm{GH}$, and in women with GH than in women with normal pregnancy, supporting our opinion that GH is a type of subclinical PE.

Until now, two factors, high BP levels and high total vascular resistance, are candidates of deterioration from GH to PE. ${ }^{20,21}$ Davis et al..$^{20}$ reported that women who developed $\mathrm{PE}$ after presenting with GH had generally higher awake and $24 \mathrm{hr}$ systolic blood pressure levels than those who remained as GH. In addition, Valensise et al. ${ }^{21}$ reported that women with presumptive mild $\mathrm{GH}$ in whom total vascular resistance was significantly high tended to develop PE. However, to date, there have been no studies confirming that the presumptive GH that changes to PE shows the increases of sFlt-1, sFlt1/PlGF ratio and sEng between, before and after the occurrence of PE. In future, we should plan a prospective cohort study with serial and frequent determinations of sFlt-1, PlGF and sEng from just after the onset of presumptive GH until delivery to elucidate whether the changes in these angiogenesis-related factors cause the deterioration of $\mathrm{GH}$ and change to PE.

In GH women with preterm birth, the level of PlGF was significantly lower than those with term birth, whereas in PE women with preterm birth, the level of PlGF was not significantly different between PE women with preterm birth and those with term birth. As the level of PlGF in GH women with preterm birth was almost similar to the level of PlGF in PE women with preterm and term births, and as the levels of sFlt-1, sFlt-1/PlGF ratio and sEng in GH women with preterm birth was almost similar to those in PE women with term birth, we speculate that GH with preterm birth might be a subclinical PE in which delivery occurred before emerging proteinuria. In GH women with preterm birth, the onset of GH was significantly earlier than in those with term birth $(30.2 \pm 1.7 v s .37 .5 \pm 0.8, P=0.001)$, whereas the maximum systolic blood pressure levels were not different between two groups (data not shown). Thus, the levels of PlGF in GH women 
may be associated with the onset of hypertension, but not the severity of hypertension.

In GH women with an SGA infant, the level of PlGF was significantly lower than those without an SGA infant. Similarly, in PE women with an SGA infant, the level of PlGF was also significantly lower than those without an SGA infant. Surprisingly, the levels of PlGF in both GH women with an SGA infant and PE women with an SGA infant were almost identical $(1.93 \pm 0.25$ vs. $1.96 \pm 0.05)$, indicating that the low level of PlGF in GH women may be risk factors for not only occurring earlier onset of hypertension, but also developing an SGA infant.

In our previous study, we established the gestational age-specific normal reference values of serum levels of sFlt-1, PlGF and the sFlt-1/ PlGF ratio; ${ }^{9}$ however, enzyme-linked immunosorbent assay for human sFlt-1 was recently modified from the old assay (DVR100; R\&D Systems) to a new assay (DVR100B; R\&D Systems). In this study, we newly established gestational age-specific normal reference values of sFlt-1, PlGF and the sFlt-1/PlGF ratio. When comparing the new reference values of sFlt-1 and the sFlt-1/PlGF ratio with the old reference values of sFlt-1 and the sFlt-1/PlGF ratio, the levels of sFlt-1 each week in the new curves increased parallel to the old curves, with almost 3- to 4-fold increase; and the sFlt-1/PlGF ratio each week in the new curves increased parallel to the old curves, with almost 2 - to 3-fold increase. PlGF in the new reference curves increased almost 1.2- to 1.3-fold compared with the old reference curves. These PlGF differences may be due to the storage condition. In the previous study, the storage of serum samples was not carefully and strictly controlled compared with the samples currently used to construct normal reference values; therefore, we think that the current reference range of PlGF is more reliable than the old reference range of PlGF.

In conclusion, our study revealed that the sFlt-1/PlGF ratio and sEng in women with h-PE and with si-PE increased more than in women with $\mathrm{GH}$, and the majority of women with $\mathrm{GH}$ showed abnormal increases of both sFlt-1/PlGF ratio and sEng, suggesting that GH may be a subclinical PE in view of serum levels of angiogenesis-related factors. However, we do not know whether the majority of presumptive GH are at early and mild stages of subclinical PE, which change to symptomatic PE with the deteriorations of angiogenesisrelated factors. To elucidate this hypothesis, a prospective cohort study with serial and frequent determinations of sFlt-1, PlGF and sEng from just after the onset of presumptive GH until delivery is warranted.

\section{ACKNOWLEDGEMENTS}

This work was supported by research grants (18591809) from the Ministry of Education, Science and Culture of Japan. We are indebted to M Yuzawa for the daily collection of blood samples and for centrifuging and storing samples.

1 Sato K. A proposal for a new definition and classification of 'Pregnancy induced Hypertension (PIH)' (2004) In Japan Society for the Study of Toxemia of Pregnancy (ed) Historical Perspective of Study of Pregnancy-Induced Hypertension in Japan 2005, pp 54-87.

2 National High Blood Pressure Education Program Working Group on High Blood Pressure in Pregnancy. Report of the national high blood pressure education program working group on high blood pressure in pregnancy. Am J Obstet Gynecol 2000; 183: S1-S22.

3 Brown MA, Lindheimer MD, de Swiet M, Van Assche A, Moutquin JM. The classification and diagnosis of the hypertensive disorders of pregnancy: statement from the International Society for the Study of Hypertension in Pregnancy (ISSHP). Hypertens Pregnancy 2001; 20: IX-XIV

4 Morikawa M, Yamada T, Yamada T, Cho K, Yamada H, Sakuragi N, Minakami H. Pregnancy outcome of women who developed proteinuria in the absence of hypertension after mid-gestation. J Perinat Med 2008; 36: 419-424.

5 Saudan $\mathrm{P}$, Brown MA, Buddle ML, Jones M. Does gestational hypertension become preeclampsia? Br J Obstet Gynaecol 1998; 105: 1177-1184.

6 Barton JR, O'brien JM, Bergauer NK, Jacques DL, Sibai BM. Mild gestational hypertension remote from term: progression and outcome. Am J Obstet Gynecol 2001; 184: 979-983.

7 Villar J, Carroli G, Wojdyla D, Abalos E, Giordano D, Ba'aqeel H, Farnot U, Bergsjø P, Bakketeig L, Lumbiganon P, Campodónico L, Al-Mazrou Y, Lindheimer M, Kramer M, World Health Organization Antenatal Care Trial Research Group. Preeclampsia, gestational hypertension and intrauterine growth restriction, related or independent conditions? Am J Obstet Gynecol 2006; 194: 921-931.

8 Levine RJ, Maynard SE, Qian C, Lim KH, England LJ, Yu KF, Schisterman EF, Thadhani R, Sachs BP, Epstein FH, Sibai BM, Sukhatme VP, Karumanchi SA. Circulating angiogenic factors and the risk of preeclampsia. N Engl J Med 2004; 350: 672-683.

9 Hirashima C, Ohkuchi A, Arai F, Takahashi K, Suzuki H, Watanabe T, Kario K, Matsubara S, Suzuki M. Establishing reference values for both total soluble Fms-like tyrosine kinase 1 and free placental growth factor in pregnant women. Hypertens Res 2005; 28: 727-732.

10 Levine RJ, Lam C, Qian C, Yu KF, Maynard SE, Sachs BP, Sibai BM, Epstein FH, Romero R, Thadhani R, Karumanchi SA, CPEP Study Group. Soluble endoglin and other circulating antiangiogenic factors in preeclampsia. N Engl J Med 2006; 355: 992-1005.

11 Ohkuchi A, Hirashima C, Matsubara S, Suzuki H, Takahashi K, Arai F, Watanabe T, Kario K, Suzuki M. Alterations in placental growth factor levels before and after the onset of preeclampsia are more pronounced in women with early onset severe preeclampsia. Hypertens Res 2007; 30: 151-159.

12 Hirashima C, Ohkuchi A, Matsubara S, Suzuki H, Takahashi K, Usui R, Suzuki M. Alteration of serum soluble endoglin levels after the onset of preeclampsia is more pronounced in women with early-onset. Hypertens Res 2008; 31: 1541-1548.

13 Venkatesha S, Toporsian M, Lam C, Hanai J, Mammoto T, Kim YM, Bdolah Y, Lim KH, Yuan HT, Libermann TA, Stillman IE, Roberts D, D'Amore PA, Epstein FH, Sellke FW, Romero R, Sukhatme VP, Letarte M, Karumanchi SA. Soluble endoglin contributes to the pathogenesis of preeclampsia. Nat Med 2006; 12: 642-649.

14 Maynard SE, Min JY, Merchan J, Lim KH, Li J, Mondal S, Libermann TA Morgan JP, Sellke FW, Stillman IE, Epstein FH, Sukhatme VP, Karumanchi SA. Excess placental soluble fms-like tyrosine kinase 1 (sFlt1) may contribute to endothelial dysfunction, hypertension, and proteinuria in preeclampsia. J Clin Invest 2003; 111: 649-658.

15 Suzuki H, Ohkuchi A, Matsubara S, Takei Y, Murakami M, Shibuya M, Suzuki M, Sato Y. Effect of recombinant placental growth factor 2 on hypertension induced by full-length mouse soluble fms-like tyrosine kinase 1 adenoviral vector in pregnant mice. Hypertension 2009; 54: 1129-1135.

16 Salahuddin S, Lee Y, Vadnais M, Sachs BP, Karumanchi SA, Lim KH. Diagnostic utility of soluble fms-like tyrosine kinase 1 and soluble endoglin in hypertensive diseases of pregnancy. Am J Obstet Gynecol 2007; 197: 28.e1-28.e6.

17 Ogawa Y, Iwamura T, Kuriya N, Nishida H, Takeuchi H, Yakada M, Itabashi K, Imura S, Isobe K. Birth size standards by gestational age for Japanese neonates. Acta Neonatologica Japonica 1998; 34: 624-632.

18 Harris EK, Boyd JC. Time-dependent reference values, In Harris EK, Boyd JC (eds) Statistical Bases of Reference Values in Laboratory Medicine. Marcel Dekker Inc: New York, 1995, pp 149-186.

19 Strevens H, Wide-Swensson D, Hansen A, Horn T, Ingemarsson I, Larsen S, Willner J, Olsen S. Glomerular endotheliosis in normal pregnancy and pre-eclampsia. BJOG 2003; 110: 831-836.

20 Davis GK, Mackenzie C, Brown MA, Homer CS, Holt J, McHugh L, Mangos G. Predicting transformation from gestational hypertension to preeclampsia in clinical practice: a possible role for $24 \mathrm{~h}$ ambulatory blood pressure monitoring. Hypertens Pregnancy 2007; 26: 77-87.

21 Valensise H, Vasapollo B, Novelli GP, Pasqualetti P, Galante A, Arduini D. Maternal total vascular resistance and concentric geometry: a key to identify uncomplicated gestational hypertension. BJOG 2006; 113: 1044-1052. 